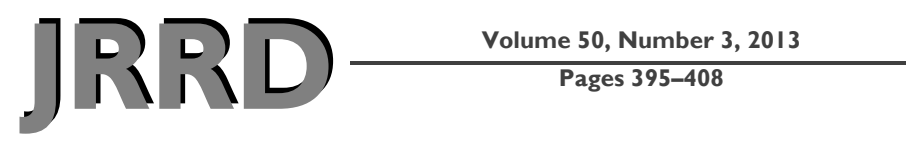

\title{
Selection of muscle and nerve-cuff electrodes for neuroprostheses using customizable musculoskeletal model
}

\author{
Dimitra Blana, PhD; ${ }^{1-2}$ Juan G. Hincapie, PhD; ${ }^{1}$ Edward K. Chadwick, PhD; ${ }^{1-2 *}$ Robert F. Kirsch, PhD ${ }^{1,3}$ \\ ${ }^{1}$ Department of Biomedical Engineering, Case Western Reserve University, Cleveland, $\mathrm{OH} ;{ }^{2}$ Department of Sport and \\ Exercise Science, Aberystwyth University, Aberystwyth, UK; ${ }^{3}$ Louis Stokes Cleveland Department of Veterans Affairs \\ Medical Center, Cleveland, $\mathrm{OH}$
}

\begin{abstract}
Neuroprosthetic systems based on functional electrical stimulation aim to restore motor function to individuals with paralysis following spinal cord injury. Identifying the optimal electrode set for the neuroprosthesis is complicated because it depends on the characteristics of the individual (such as injury level), the force capacities of the muscles, the movements the system aims to restore, and the hardware limitations (number and type of electrodes available). An electrode-selection method has been developed that uses a customized musculoskeletal model. Candidate electrode sets are created based on desired functional outcomes and the hardware limitations of the proposed system. Inverse-dynamic simulations are performed to determine the proportion of target movements that can be accomplished with each set; the set that allows the most movements to be performed is chosen as the optimal set. The technique is demonstrated here for a system recently developed by our research group to restore whole-arm movement to individuals with high-level tetraplegia. The optimal set included selective nerve-cuff electrodes for the radial and musculocutaneous nerves; single-channel cuffs for the axillary, suprascapular, upper subscapular, and long-thoracic nerves; and muscle-based electrodes for the remaining channels. The importance of functional goals, hardware limitations, muscle and nerve anatomy, and surgical feasibility are highlighted.
\end{abstract}

Key words: functional electrical stimulation, muscle electrode, musculoskeletal model, nerve-cuff electrode, neuroprosthesis, rehabilitation, shoulder, simulation, spinal cord injury, upper limb.

\section{INTRODUCTION}

Neuroprostheses restore motor function after spinal cord injury (SCI) by applying functional electrical stimulation (FES) to paralyzed muscles. The number of muscles targeted by the FES system is a function not only of the level of injury, which determines the extent of the paralysis, but also of the number of stimulation channels available in the neuroprosthesis itself. For example, arm function in low tetraplegia (cervical [C]7 SCI) can be significantly improved with stimulation of just the triceps [1], because most of the other arm muscles are under voluntary control. However, in the same population, all hand muscles are typically paralyzed and FES systems that restore basic hand function (i.e., grasp and release) use at least eight stimulating channels [2]. Restoring more natural and dexterous hand function would require many more channels than the current neuroprostheses can provide [3]. In this case, it is the availability of stimulating channels

\footnotetext{
Abbreviations: $\mathrm{C}=$ cervical, $\mathrm{EMG}=$ electromyography, $\mathrm{FES}=$ functional electrical stimulation, LED = light emitting diodes, SCI = spinal cord injury.

*Address all correspondence to Edward K. Chadwick, PhD; Institute for Science and Technology in Medicine, Guy Hilton Research Centre, Keele University, Thornburrow Drive, Stoke-on-Trent, ST4 7QB, UK; +44-1782-554600.

Email: e.k.j.chadwick@keele.ac.uk

http://dx.doi.org/10.1682/JRRD.2012.02.0034
} 
and not the remaining motor function that limits the number of muscles targeted for FES.

As the number of available stimulating channels increases, the problem of determining which muscles to stimulate to restore the most function to a specific individual becomes both difficult and important for the success of the FES system. One way to solve this problem is to identify the appropriate muscle groups based on their known functions and choose a subset of these according to their surgical accessibility and level of response to electrical stimulation. In the case of the hand grasp system just mentioned, a limited group of primary muscles for hand function was chosen and supramaximal surface stimulation was used to exclude muscles with complete denervation [3]. Similarly, in the design of lower-limb FES systems for standing, the target muscles were chosen based on biomechanical analysis, feedback from other users, and prior testing with surface and percutaneous electrodes [4]. These methods work well in systems that aim to restore a limited number of functions, such as simple hand grasp or a fixed standing pattern, but not in systems that target a wider range of movements. A more complex example is restoration of shoulder and arm function to individuals with complete upper-limb paralysis due to high tetraplegia (C1-C4 SCI). This kind of system aims to restore many different movements (e.g., reaching to the front or the side, eating); therefore, different sets of muscles are important in each case. Moreover, many muscles do not have obvious effects on the movement of limb segments, but rather act to stabilize joints (e.g., the rotator cuff muscles stabilize the glenohumeral joint). Finally, feedback from previous users is limited, because FES applications to individuals with high tetraplegia have been very limited in number and based on temporary surface [5-6] or percutaneous [7] stimulation. Furthermore, in these studies, the stimulation patterns were based on nondisabled electromyography (EMG) patterns, which do not consider partial muscle denervation and disuse atrophy, and relied upon external support of the weight of the arm. For these reasons, the optimal muscle set for stimulation in high tetraplegia cannot be identified by using only previous experience and biomechanical observations.

A different approach involves the use of a musculoskeletal model to investigate the likely effect of different stimulating electrodes on limb function. Using a model during the initial development of a neuroprosthesis means that a large number of electrode configurations can be tested before human implementation, thus avoiding time-consuming and potentially invasive trial-anderror procedures on the FES participant. Kirsch et al. used the shoulder model developed by van der Helm [8] to investigate the effect of the stimulation of various shoulder muscles on arm mobility and stability, both in midcervical level (C5-C6) and high-level (C1-C4) tetraplegia [9]. More recently, the same upper-limb model was used by Hincapie et al. to determine that stimulation of the serratus anterior, infraspinatus, and triceps can restore a large range of movements in the midcervical SCI population [10].

The studies just mentioned aimed to identify the optimal muscle set for the specific level of SCI, but in order for a neuroprosthesis to be feasible, additional hardware considerations need to be taken into account. Two types of implanted electrodes are currently available for an actual neuroprosthesis and their different effects need to be included in the model simulations: muscle-based and nerve-cuff electrodes. Muscle-based electrodes are placed on the surface of a muscle [11] or inserted into a muscle [12] and activate nerve branches in the immediate vicinity of the electrode. They are most effective for smaller (e.g., hand) or physically isolated (e.g., pectoralis major) muscles. Nerve-cuff electrodes wrap around a peripheral nerve before they reach a muscle and activate all the muscle fibers innervated by that nerve [13]. This makes them particularly useful for shoulder muscles that are too broad to be activated by a single muscle electrode (e.g., the nine-compartment serratus anterior), for muscles that might sustain damage due to the electrode sliding against bones during movement (e.g., deltoid), or for muscles that are deep or otherwise represent difficult surgical targets (e.g., the rotator cuff muscles). In addition, if multiple muscles are innervated by the same nerve, the nerve cuff may either activate all of them or, if it has multiple contacts, selectively activate different muscles [14]. Polasek et al. demonstrated the feasibility of selective activation of muscles in the upper limb by using spiral nerve-cuff electrodes with four contacts [15]. Either single-contact activation or activation of adjacent contacts was used to achieve selective activation of either the triceps or extensor digitorum muscles (in the case of the radial nerve) or the biceps brachii or the brachialis muscles (in the case of the musculocutaneous nerve). Activation of a single muscle up to 48 percent was achieved before coactivation of another muscle innervated by the same nerve occurred using a single contact. With "current 
steering" induced by multiple contact activation within the same cuff, this percentage increased.

The exact location of the nerve-cuff electrode along the nerve is another consideration, because different parts of the nerve could be surgically suitable for nerve-cuff electrode placement. As the nerves of the shoulder and arm leave the brachial plexus, they give off branches that innervate different muscles. Figure 1 shows a schematic of the radial nerve, which originates in the posterior cord of the brachial plexus, runs down the posterior and then anterior side of the upper arm, and continues in the forearm, where it branches into a superficial and deep branch.

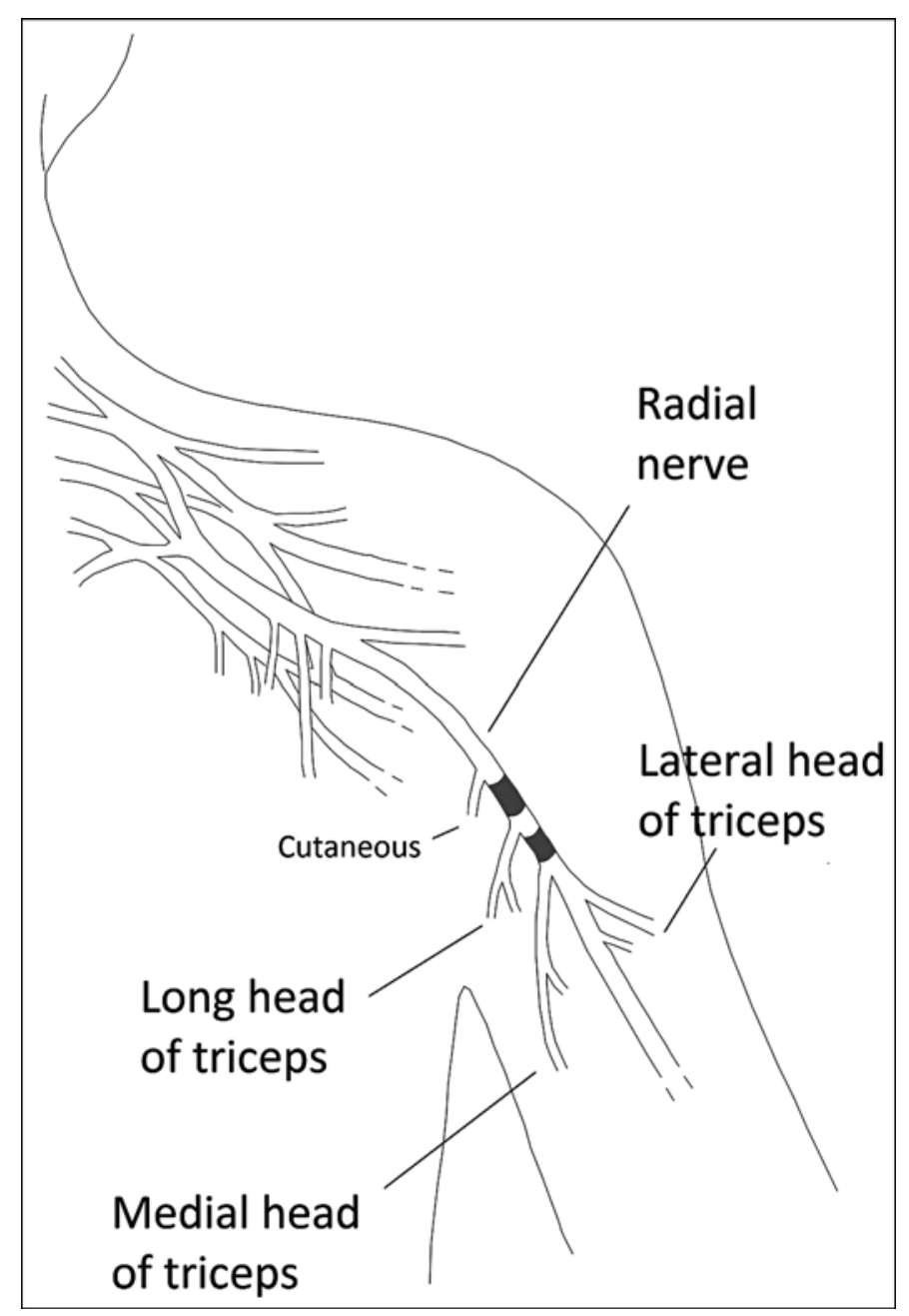

Figure 1.

Posterior view of brachial plexus and course of radial nerve in upper arm. Shown are branches to long, medial, and lateral heads of triceps and two possible locations for nerve-cuff electrode: right before and right after branch to long head of triceps.
Along the way, it gives off branches to the long head of the triceps, the medial and then lateral head of the triceps, the brachialis, brachioradialis and, finally, wrist and finger muscles. Two different surgically accessible nervecuff placement locations are shown in Figure 1: either before or after the branch to the long head of the triceps, which would result in the triceps long head being stimulated or not by the cuff. Another example is the serratus anterior, a large muscle originating on the ribs and inserting on the scapula, innervated by the long thoracic nerve. Hincapie et al. showed that it is optimal to stimulate only the lower half of this muscle, because that is the part that keeps the scapula against the thorax and prevents scapular "winging" [10]. This can be surgically achieved by placing a nerve-cuff electrode on the lower half of the long thoracic nerve.

In this study, we present an electrode-selection method that (1) uses a customizable musculoskeletal model instead of trial-and-error experimentation to objectively evaluate the mechanical and functional effect of different electrode sets before human implementation and (2) considers the surgical accessibility and the types and number of electrodes available in the proposed neuroprosthesis to ensure that the optimal electrode set is also feasible. The method is demonstrated in a neuroprosthesis for restoration of whole-arm functional movements that was developed by our research group and recently implemented in a human subject with high-level tetraplegia.

\section{METHODS}

\section{Neuroprosthesis}

The first step in the selection of the optimal electrode set for FES is a clear description of the requirements and hardware constraints of the FES system itself. In our case, it was the first fully implanted FES system that aimed to restore shoulder, arm, and hand movement to people with high-level tetraplegia. It consisted of two implantable stimulator-telemeters [16], each with 12 stimulating channels and 2 EMG recording channels, for a total of 24 stimulation channels. The 10 channels used for hand-grasp control were not chosen by a model-based method, but rather were based on extensive previous experience [3]. However, a model-based approach was necessary for the remaining 14 channels reserved for shoulder and arm muscle stimulation, since there was no previous experience of whole-arm FES control. For these 
channels, an initial list of possible nerves (for nerve-cuff electrodes) and muscles (for muscle-based electrodes) was drawn up, taking surgical accessibility into account. This list is shown in the first column of Table 1. It includes possible nerve-cuff electrodes on the radial (triceps, brachialis, brachioradialis, wrist, and finger muscles), musculocutaneous (biceps, brachialis, brachioradialis), axillary (deltoid and teres minor), suprascapular (infraspinatus and supraspinatus), subscapular (subscapularis), and long thoracic (serratus anterior) nerves and intramuscular electrodes for the rhomboid, upper and lower pectoralis major, pronator quadratus, and supinator. Some of the nerve-cuff electrodes have more than one placement option: the cuff on the radial nerve can be placed before or after the branch to the long head of the triceps (as shown in Figure 1), the cuff on the musculocutaneous can include or avoid the coracobrachialis, and similarly, the cuff on the axillary nerve can include or avoid the teres minor. For the long thoracic nerve that innervates the serratus anterior, a previous study already showed that the cuff is most beneficial if it only stimulates the lower half of the muscle [10].

\section{Model}

The model used in this study was built in Software for Interactive Musculoskeletal Modeling (MusculoGraphics, Inc; Santa Rosa, California), a graphics-based system developed especially for musculoskeletal modeling. The model consists of six bones (sternum, clavicle, scapula, humerus, ulna, and radius) and five joints (sternoclavicular, acromioclavicular, glenohumeral, humeroulnar, and radioulnar). The three joints at the shoulder are ball joints, with 3 degrees of freedom each, while the elbow and forearm joints are 1-degree-of-freedom, single-axis joints. The model also includes the scapulothoracic gliding plane [8], which means that the medial border of the scapula is constrained to maintain contact with the thorax. The muscles are represented by one or more contractile elements, depending on their size and the width of their attachment site [8]. The use of multiple contractile elements for a single muscle reflects the sometimes significantly different lines of actions of different fibers within the muscle. Thus, the model includes 29 muscles but a total of 138 contractile elements. The model parameters were obtained from cadaver studies by Klein-Breteler et al. [17]. These parameters include the position of joint centers,

Table 1.

Initial and final electrode sets. First column shows nerve and muscle candidates for stimulation by high-tetraplegia functional electrical stimulation system, which were chosen from muscles of upper limb based on functional considerations and surgical accessibility. Second column shows electrodes assigned to each nerve/muscle by our selection algorithm. This is electrode set that had highest simulation success rate in 10 movements of interest. Second column also specifies number of contacts in case of selective cuff electrodes. Third column shows placement of electrodes on nerves, when important.

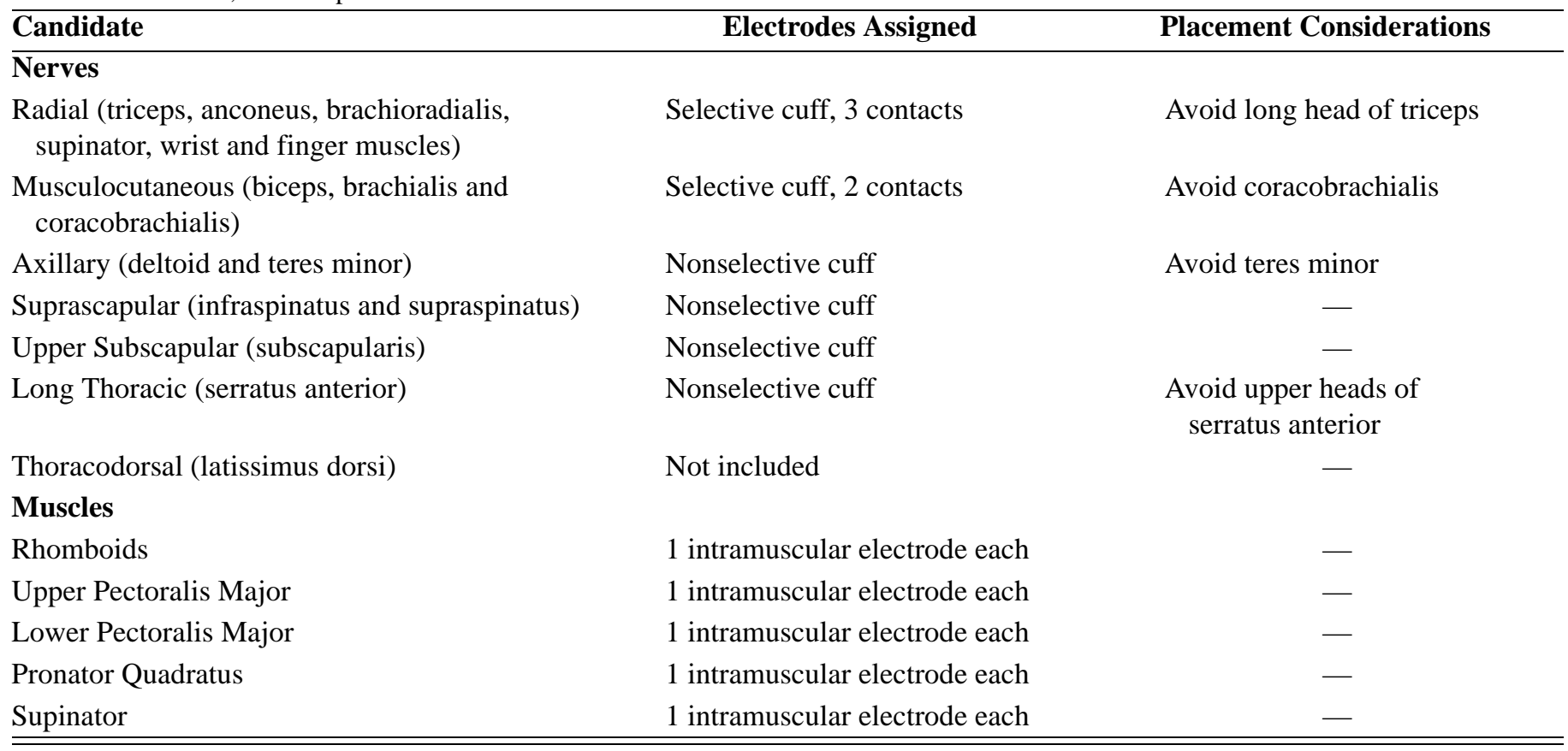


inertial parameters for body segments; the number of elements representing each muscle; and the optimal fiber length, origin and insertion, tendon slack length, physiological cross-sectional area, and pennation angle of every element. A detailed description of the model and its evaluation can be found in Blana et al. [18].

The model was customized to simulate a person with a complete high-level SCI. An injury at high cervical levels causes paralysis of almost all upper-limb muscles, with the usual exception of the levator scapulae and upper trapezius [9]. To simulate a subject with high-level SCI, all other muscles were set to have zero active force, and in addition, the maximum forces for the levator scapulae and upper trapezius were set to 50 percent of nondisabled maximum force to simulate the effects of possible muscle denervation and partial paralysis [18].

For the muscles that would be stimulated by the neuroprosthetic system using muscle or nerve-cuff electrodes, the maximum forces were set to 50 percent of the average nondisabled maximum forces [19]. This reduction simulates partial muscle denervation and, in the case of muscle electrodes, limited spatial muscle activation due to electrode placement. The effects of FES were further simulated by constraining all the multiple elements representing a stimulated muscle in the model to have the same level of activation. For example, the rhomboid, a diamond-shaped muscle that originates on the spine and attaches on the medial border of the scapula, is represented in the model by five elements. If we assume that the muscle is stimulated by a single electrode, then during the model simulations, all five elements are required to have the same activation level.

In the case of nonselective nerve-cuff electrodes, we further assumed that all muscles innervated by the nerve distal to the nerve-cuff electrode placement have the same level of activation. Thus, if we placed a nonselective nerve-cuff electrode on the radial nerve (see Figure 1) prior to branching to the various muscles, we would require all the elements that represent the long, medial, and lateral head of the triceps, brachialis, and brachioradialis to have the same activation level. (The model does not include the wrist and hand, otherwise the wrist and hand muscles innervated by the radial nerve would have the same activation as well.) It is obvious that because the radial nerve innervates both flexors (brachialis, brachioradialis) and extensors (triceps) of the elbow, a nonselective nerve-cuff electrode would not be a good solution.
To summarize, the model was customized to the characteristics of the FES participant by reducing the maximum force the muscles can produce depending on whether they are under voluntary control, paralyzed and not under FES stimulation, or paralyzed and stimulated by FES. The model was also modified to simulate different types and placements of electrodes by constraining muscle elements, or entire groups of muscles to have the same activation level, and including or excluding muscles from stimulation according to their source of innervation.

\section{Simulations}

The model was used here to run inverse-dynamic simulations including different sets of stimulating electrodes that activated different sets of muscles. A diagram of the model including the steps of an inverse-dynamic simulation is shown in Figure 2. The inputs to these simulations are arm movements expressed as patterns of joint angles, and the outputs are the required muscle forces and activations that allow these arm movements to be performed. Muscle activations are approximated in the model by dividing each muscle force by the maximum force that muscle can produce at the specified arm configuration.

Using inverse dynamics, the required torques at each joint are calculated from the input joint angles. Since the model includes more muscles than degrees of freedom, a nonlinear constrained optimization routine is used to resolve these torques into individual muscle forces. The optimization aims to minimize muscle energy consumption, using a cost function proposed by Praagman et al. [20]. This method showed good correspondence with experimental muscle activity approximated using surface EMG. The optimization includes three types of constraints: the muscle forces are constrained to produce the torques required by the input movement; the muscle forces acting on the glenohumeral joint are constrained to pull the humerus toward the glenoid cavity, ensuring stability of the joint; and the muscle forces acting on the scapula are constrained to keep the scapula pressed against the thorax in order to avoid scapular winging.

At each movement time step, the model either succeeds and reports the required muscle forces and activations or fails to find a successful solution and reports the reason for failure. Failure is due to violation of one of the three types of constraints previously mentioned: insufficient muscle force to generate the required moments at each joint, inability to maintain glenohumeral stability, or inability to avoid scapular winging. 


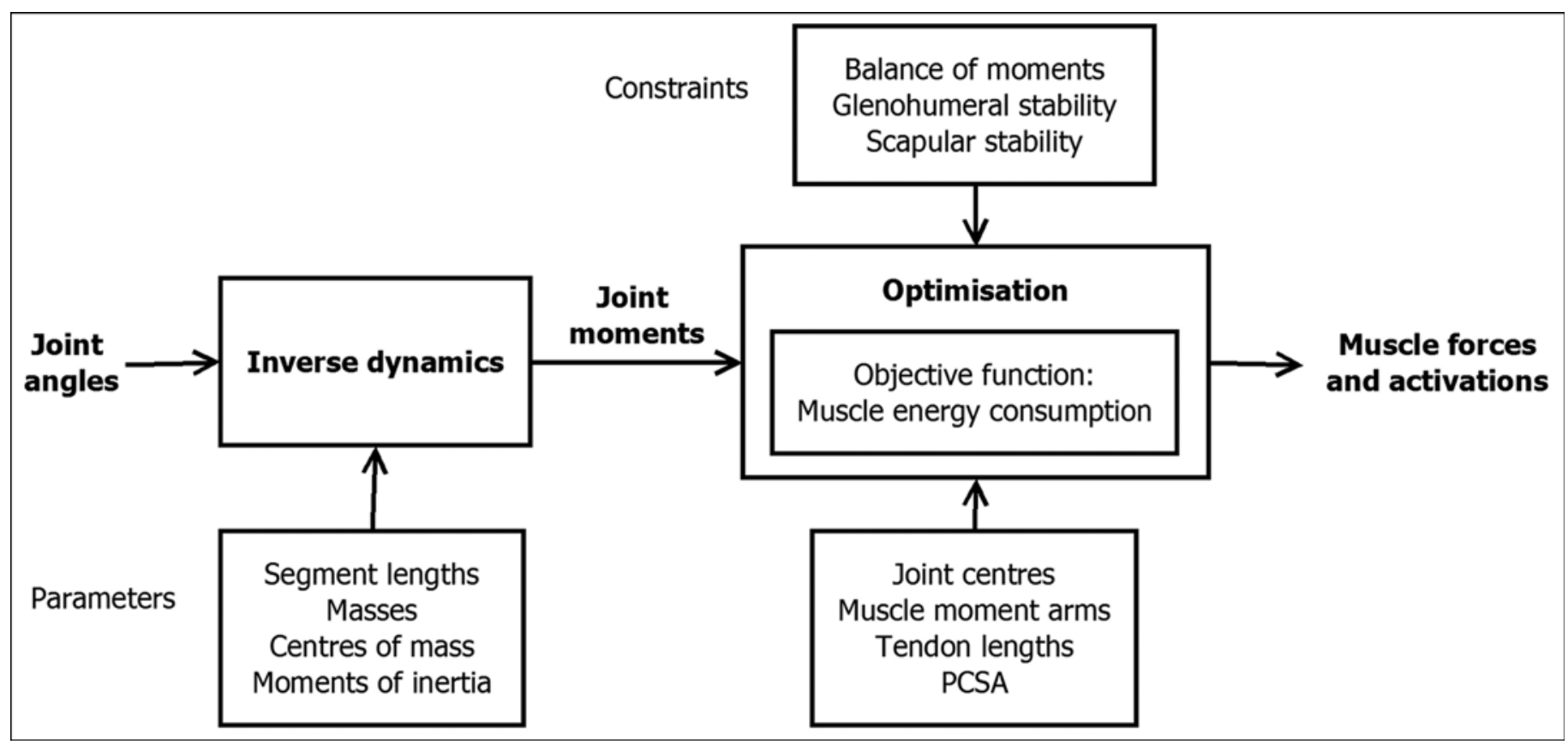

\section{Figure 2.}

Block diagram of model. Inputs are 11 angles of shoulder and elbow (3 each at sternoclavicular, acromioclavicular, and glenohumeral joints; elbow flexion-extension; and forearm pronation-supination). Using model parameters such as mass and center of mass of every segment, inverse dynamics calculates the required torques at each joint from input joint angles. Individual muscle forces are then calculated using optimization method that aims to minimize energy consumption under 3 sets of constraints: balance of moments, glenohumeral stability, and scapular stability. To accurately model force-production capacity of muscles, optimization uses parameters such as tendon slack length and muscle physiological cross-sectional area (PCSA). Final output of model is force and activation of each muscle. Activation is approximated by dividing force by maximum force muscle can produce at specified arm configuration.

For each movement, the success rate $S R$ is defined as the ratio of the number of successful time steps to the total number of time steps for that movement (Equation (1)):

$$
S R=\frac{\text { Successful Time Steps }}{\text { Total Time Steps in Movement }} \quad \text {. }
$$

Figure 3 shows an example of a fully successful and a partially successful simulation of a simple arm elevation movement. The elevation angle that the model is aiming to achieve is shown in Figure 3(a). Figure 3(b) shows the activation patterns of the three heads of the deltoid, which are outputs of a simulation that included all candidate muscles (shown in the first column of Table 1) individually activated, a "best-case" scenario. Every step of this simulation was successful. The simulation shown in Figure 3(c) included a nonselective cuff electrode on the axillary nerve, which explains why there is only one activation pattern for the entire deltoid muscle. This con- straint resulted in the inability to generate the required elevation moment for a large part of the simulation (gray bands in 3(c), elevation above $65^{\circ}$ ). A comparison between 3(b) and 3(c) shows that failure occurred when the successful activation patterns of the three deltoid heads were most different; this was not allowed by the nonselective cuff.

In this study, 10 different movements were simulated: humeral elevation in three planes (frontal, sagittal, scapular), elbow flexion-extension, forearm pronationsupination, and the following simulated functional movements: reaching to three different heights, "eating” and "combing the hair." Thus, this set contains a sample of functional activities as well as single-axis movements that, as components of more complicated motions, need to be themselves feasible.

The movements used as inputs to the inverse kinematic simulations were recorded from one nondisabled subject after giving informed consent. Data were recorded 
(a)

$(\circ)$

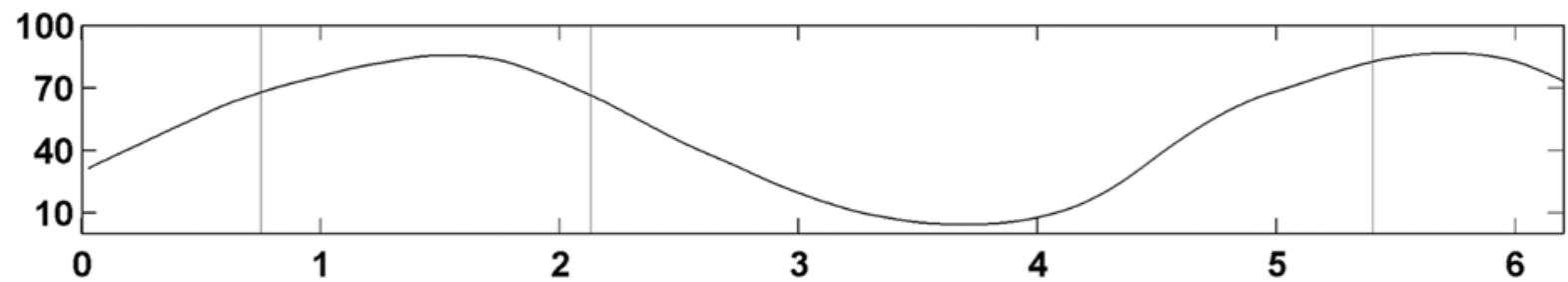

(b)

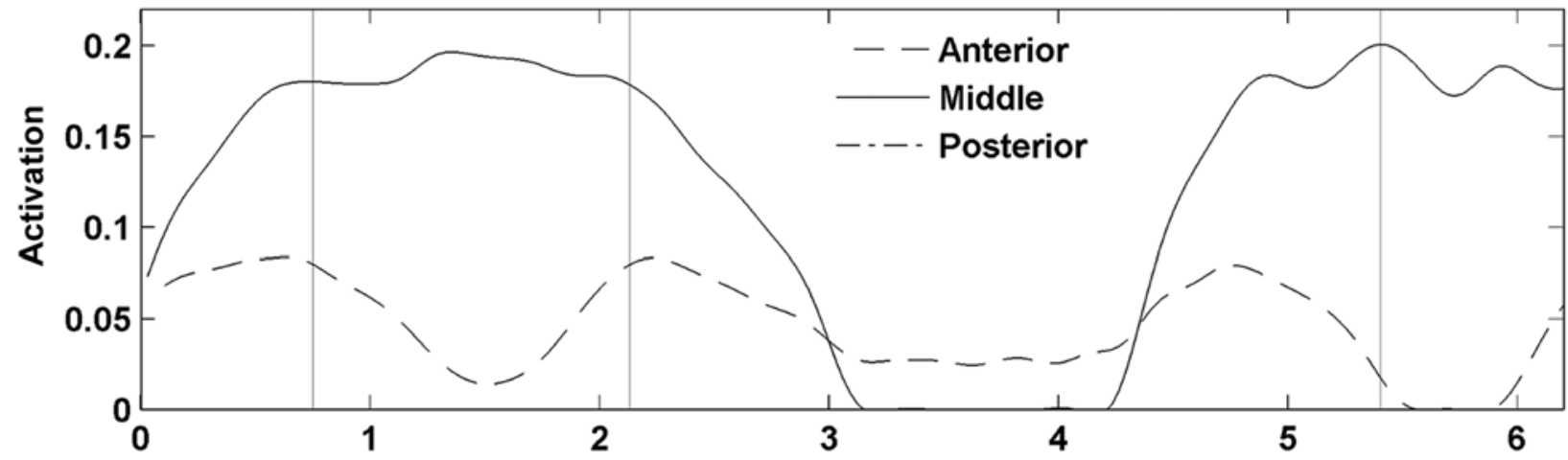

(c)

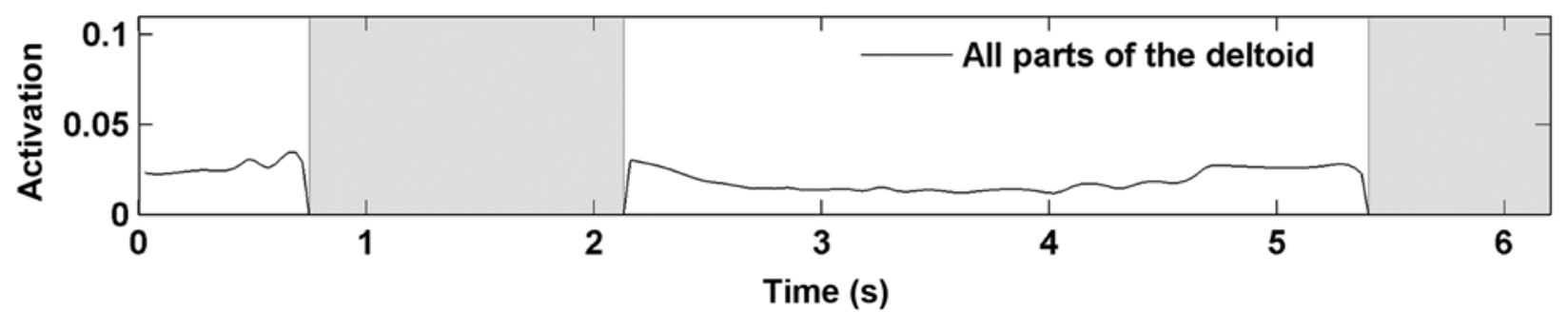

Figure 3.

Example of fully successful and partially successful simulation of simple arm elevation. (a) Elevation angle that model is aiming to achieve (model input). (b) Activation patterns of 3 heads of deltoid, which are outputs of simulation that included all candidate muscles individually activated. (c) Simulation included nonselective cuff electrode on axillary nerve, where activations of different heads of muscle are constrained to be the same. Gray bands show time steps for which simulation was unsuccessful, with activations constrained in this way.

using an Optotrak system (Northern Digital Inc; Ontario, Canada), which includes three cameras that trace the three-dimensional positions of light emitting diodes (LED) located within the workspace. In order to trace the position of the thorax, humerus, and forearm of the subject, sets of LED were fixed over these segments and their locations were recorded at $30 \mathrm{~Hz}$. Because the locations of the scapula and clavicle are difficult to accurately measure during movements, the locations of the torso, arm, clavicle, and scapula were recorded using a palpation technique for a number of static postures throughout the workspace. A three-dimensional regression model was then built to relate static humeral positions to static scapular and clavicular positions, and this model was used to predict dynamic scapular and clavicular positions from dynamic humeral position, as has been done in previous studies $[10,21]$.

\section{Selection Algorithm}

As mentioned previously, the various peripheral nerves (for nerve-cuff electrodes) and muscles (for muscle-based electrodes) that are candidates for stimulation are presented in the first column of Table 1. In order to reduce the number of candidate sets, the first step was to determine which of these muscles and nerves were absolutely essential for the simulated movements. This was 
done using the following method. A "best-case" scenario set was created that included all the candidate nerves and muscles individually stimulated. Inverse-dynamic simulations were run, and the success rate of this set was calculated for each of the 10 movements. Then, one candidate at a time was removed from this set, and the simulations were run again. If, with the exclusion of the candidate, the success rate for all movements did not fall below a certain threshold compared with the best-case success rate, the candidate was considered nonessential.

Here, we defined the threshold as 50 percent of the success rate of the best-case scenario set. The best-case success rate is not necessarily 100 percent, because the SCI-adjusted model includes a number of muscles that were not targeted for FES and even those targeted for FES have reduced maximum muscles forces. Thus, the success rate of the best-case scenario, especially for movements that require high levels of muscle force (e.g., reaching for something at shoulder level) is expected to be low. If the best-case success rate for movement $i$ is $\max S R_{i}$, and the success rate of a simulation of the same movement that does not include muscle or nerve $j$ is $S R_{i j}$, then $j$ was considered nonessential if (Equation (2)) -

$$
\text { Relative } S R_{j}=\frac{S R_{i j}}{\max S R_{i}}>0.5,
$$

for $i=1$ :total number of movements.

Once the essential muscles and nerves (i.e., those with relative $S R_{j}<0.5$ for at least one movement) were identified, a second set of simulations was run to determine which nonessential muscles should be stimulated to maximize performance with the number of remaining stimulation channels. These simulations also examined the best options for the nerve-cuff electrodes, i.e., whether they should be placed proximally or distally to a muscle branch and which ones should be selective. Because of the limited number of stimulation channels in our neuroprosthetic system (14 for shoulder and arm muscles), we decided to include two selective nerve-cuff electrodes and as many nonselective cuffs as necessary. One of the selective cuff electrodes was reserved for the radial nerve because it innervates both extensors (triceps) and flexors (brachialis, brachioradialis) of the elbow, as well as wrist and finger muscles, providing opportunities for restoring multiple functions from a single cuff while reducing the likelihood of unintended activation of multiple muscles. The remaining selective cuff was evaluated on all other targeted nerves that innervate more than one muscle (i.e., musculocutaneous, axillary, suprascapular). Based on these different options, all possible electrode sets were created and inverse-dynamic simulations were run by the model. The set with the highest mean success rate across all 10 movements was chosen as the targeted set of the neuroprosthetic system.

\section{RESULTS}

Figure 4 summarizes the results of the first set of 120 simulations, which were focused on identifying the "essential" nerves and muscles for stimulation. The column on the right (maxSR) shows the success rate for each of the different movements for the best-case scenario, i.e., the set of muscles that included all the candidate muscles individually stimulated and all the candidate nerves stimulated selectively. As expected, the simulated FES system for an SCI-adjusted model could restore only a fraction of the nondisabled movements. For example, the maxSR was as low as 0.53 for the movement that simulated "combing hair."

The rest of Figure 4 illustrates the effects on the success rate when every muscle or nerve was removed one at a time from the best-case scenario set. The gray scale from black to white corresponds to a relative success rate from 0 to 1 . It is clear that the relative success rate of certain movements was near zero (black shading) if certain individual electrodes were removed: the pronator quadratus and supinator were essential for forearm pronation/ supination; the pectoralis major was essential for elevation in the sagittal and scapular planes; the nerve cuffs on the long thoracic, radial, and musculocutaneous nerves were essential for all movements. Three electrodes were considered nonessential and are shown on the right of the figure: the muscle electrode on the rhomboid and the nerve cuffs on the thoracodorsal and subscapular nerves.

The essential set includes all electrodes that are definitely part of the optimal set. However, nonessential electrodes could also be included in the optimal set if they have a positive contribution and are permitted based on the hardware limitations of the system (i.e., the number of available electrodes). The next step, therefore, was to create candidates for the optimal electrode set by including all possible combinations of the nonessential electrodes $\left(2^{3}=8\right.$ sets). The number of candidate sets was then expanded based on the nerve-cuff electrode options mentioned earlier (i.e., selective, nonselective, placed 


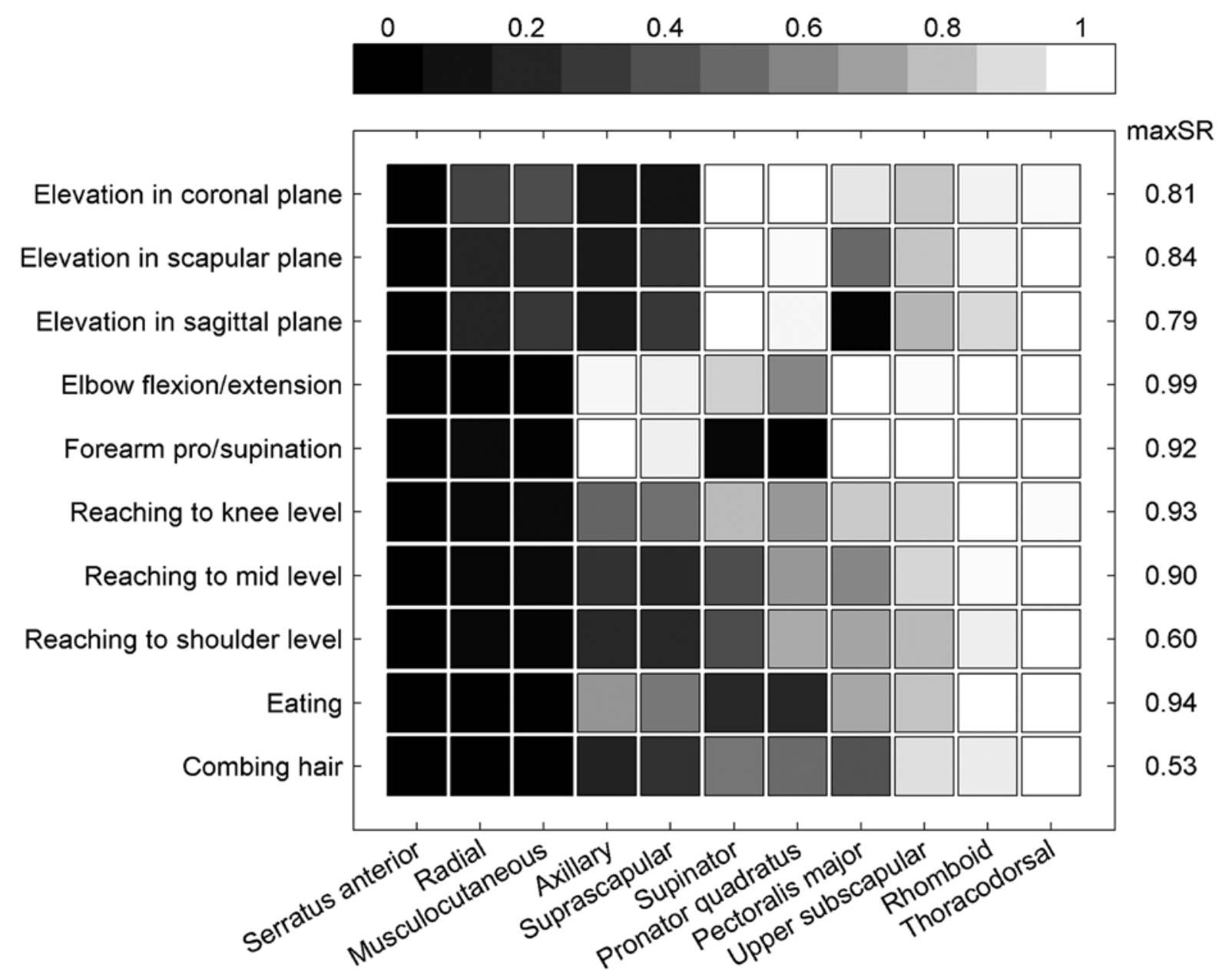

Figure 4.

Relative success rate of different electrode sets for every simulated movement. Column on right shows success rate of best-case scenario (maxSR), which consists of all candidate muscles or nerves individually stimulated. Rest of figure shows best-case scenario set, excluding 1 muscle or nerve at a time. Black corresponds to success rate of 0 and white to success rate of 1 , relative to best-case-scenario success rate. pro = pronation.

proximally or distally to a muscle branch). The total number of sets was 1,024 . When the number of available electrodes in the system (14) was taken into account, the number of candidate sets was reduced to 260 . These sets were used in simulations with all 10 movements as inputs, and the success rate was calculated for each one. The optimal electrode set was the one with the highest mean success rate, and it is shown in the second column of Table 1. This set includes two selective nerve-cuff electrodes, four nonselective nerve-cuff electrodes, and five muscle-based electrodes. In addition, the nerve-cuff channels include specific locations along several of the peripheral nerves that are distal to the nerve branches for undesirable muscles. The optimal radial nerve-cuff location was found to be distal to the nerve branch to the long head of triceps, the optimal axillary nerve-cuff location distal to the nerve branch to the teres minor, and the optimal musculocutaneous nerve-cuff location distal to the branch to the coracobrachialis. Table 2 shows the success rate of this optimal electrode set for all the various simulated movements. The mean success rate across all movements was 0.40 .

As noted, we had reserved one selective cuff electrode for the radial nerve. The simulation results were used to choose one selective cuff electrode from among the axillary, musculocutaneous, and suprascapular nerves, 
JRRD, Volume 50, Number 3, 2013

Table 2.

Success rate of optimal electrode set for every simulated movement and mean success rate.

\begin{tabular}{lc}
\hline \multicolumn{1}{c}{ Movement } & Success Rate \\
\hline Humeral Elevation in Coronal Plane & 0.27 \\
Humeral Elevation in Scapular Plane & 0.15 \\
Humeral Elevation in Sagittal Plane & 0.13 \\
Elbow Flexion-Extension & 0.82 \\
Forearm Pronation-Supination & 0.76 \\
Reaching to Knee Level & 0.65 \\
Reaching to Mid Level & 0.47 \\
Reaching to Shoulder Level & 0.15 \\
Eating & 0.49 \\
Combing Hair & 0.13 \\
Mean & 0.40 \\
\hline \hline
\end{tabular}

with the remaining two nerves receiving nonselective cuff electrodes (along with the long thoracic and subscapular nerves, which were predetermined to receive nonselective cuffs because each nerve serves only a single muscle, serratus anterior and subscapularis, respectively). Figure 5 shows the success rates when each of the three different nerves was selected as the remaining selective cuff. The illustrated success rate for each nerve was obtained using the best muscle set (i.e., the one with the highest mean success rate) from all the tested muscle sets that included the indicated nerve. The black bars indicate the success rate when the axillary nerve cuff was selective, the gray bars when the musculocutaneous nerve cuff was selective, and the white bars when the suprascapular nerve cuff was selective. Although use of a selective axillary nerve cuff provided the highest success rate for humeral elevation in all planes, the use of a selective musculocutaneous cuff electrode provided much greater success rates for almost all other movements. The use of a selective suprascapular nerve cuff did not produce a higher success rate for any of the movements examined. The overall average success rate for the selective musculocutaneous nerve cuff $(0.40)$ was nearly twice that of the selective axillary nerve (0.21) and more than three times that of the selective suprascapular nerve cuff $(0.12)$.

\section{DISCUSSION}

This study aimed to demonstrate the use of a musculoskeletal model to identify the optimal nerve and muscle set for stimulation by an FES system. Even a state-of-theart system would not have the capacity to stimulate all paralyzed muscles; therefore, a choice needs to be made about how best to select and activate the limited set of muscles available to achieve the best function. This approach was demonstrated for a specific system that aims to restore upper-limb motor function in individuals with high tetraplegia. The consequences of a high-level injury on muscle function, as well as the application of FES using different electrode types, were simulated in the model as realistically as possible using constraints on the muscle forces and activation patterns. Moreover, practical constraints such as the number of stimulating channels available in the proposed neuroprosthesis and anatomical constraints regarding the feasible electrode positions were taken into account to propose a nerve and muscle set that would be a reasonable target for the FES system.

The set identified as the optimal set included "prime movers" of the shoulder (deltoid, pectoralis major), muscles that stabilize the shoulder (infraspinatus, supraspinatus, subscapularis, serratus anterior, rhomboids), muscles for elbow flexion-extension (biceps, brachialis, medial and lateral triceps), and muscles for forearm pronationsupination (pronator quadratus, supinator). This muscle set had a relatively high success rate for simple movements of the elbow and forearm (0.76-0.82) and reaching to a low-level target such as a tabletop (0.65). The success rate was the lowest $(0.13-0.15)$ for movements that require significant humeral elevation, like combing hair or reaching for something at shoulder level. This results from the mechanical actions of the nonselective cuff on the axillary nerve that activates all three heads (anterior, middle, and posterior) of the deltoid at the same level. The deltoid is a wide muscle, and even though its primary action is humeral elevation, the lowest fibers of the anterior and posterior part actually pull the humerus downwards. Another effect of the nonselective cuff for the deltoid is that movement in the sagittal plane, which requires more anterior than posterior deltoid, is degraded. This can be seen in Table 2 by comparing the success rate for elevation in the sagittal plane (0.13) and coronal plane (0.27).

A selective nerve cuff on the musculocutaneous nerve was shown to be critical for elbow control, which is important for many activities of daily living, such as eating. This is shown in Figure 5, where a nonselective cuff on the musculocutaneous nerve resulted in success rates for elbow flexion-extension and eating of less than 


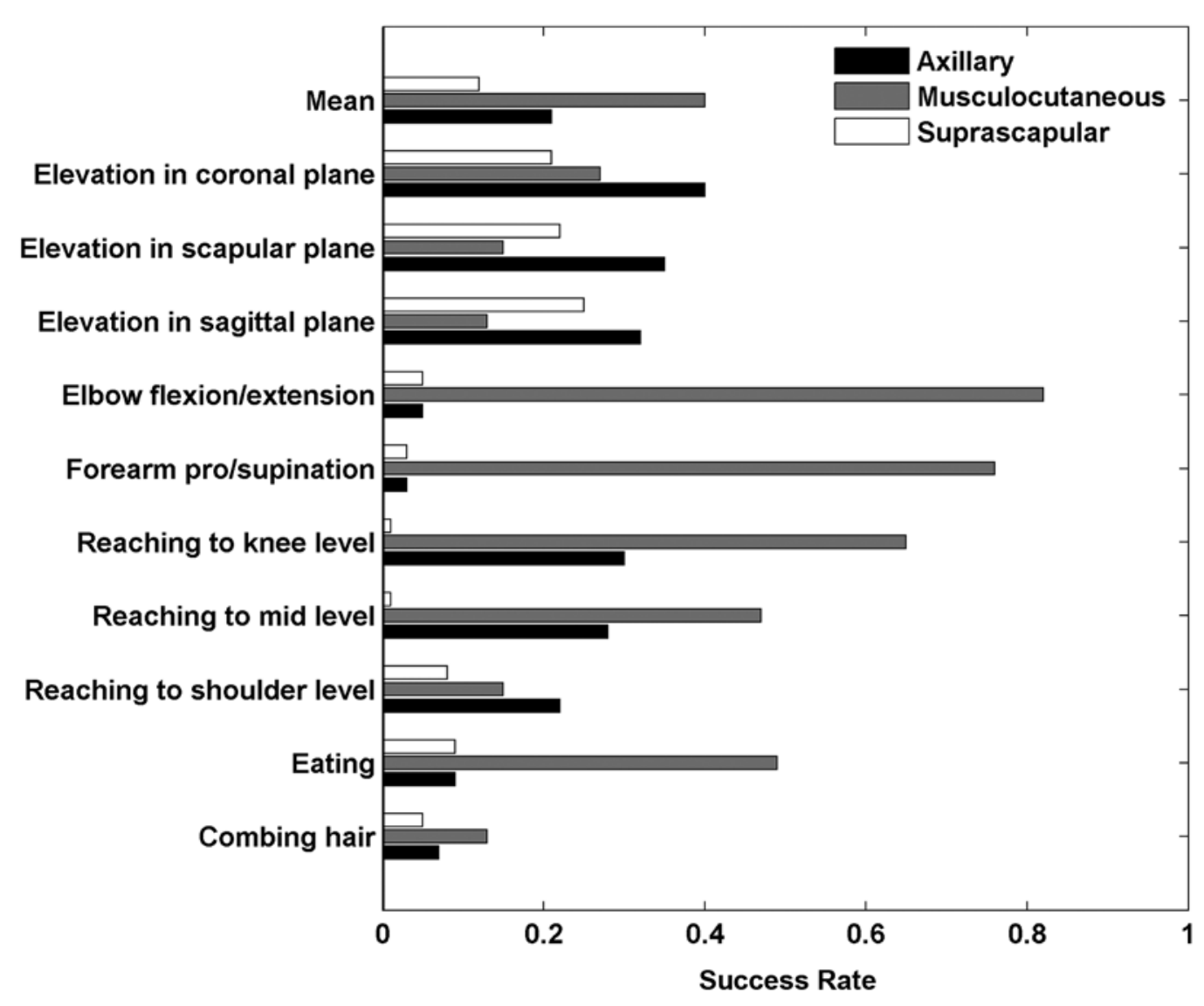

Figure 5.

Effect of different selective and nonselective nerve-cuff electrodes. Radial nerve was stimulated with selective cuff in all cases, but there were 3 options for second selective cuff: axillary, musculocutaneous, or suprascapular nerve. Black bars show simulation success rates when selective cuffs were placed on radial and axillary nerves, gray bars show success rates when selective cuffs were placed on radial and musculocutaneous nerves, and white bars show success rates when selective cuffs were placed on radial and suprascapular nerves. pro = pronation.

0.10. The biceps and brachialis are both elbow flexors, but the biceps also acts on the scapula (for humeral flexion) and on the radius (for forearm supination). The simulations showed that the difference in muscle action between biceps and brachialis was so significant that effective control of the elbow was largely prevented by their simultaneous activation.

Cuff electrode placement was found to be another important factor for the success of the simulated movements. The teres minor causes external rotation of the humerus, which is not always desirable during abduction movements performed by the deltoid. As a result, the optimal axillary nerve cuff location was found to be distal to the teres minor. Similarly, the long head of the triceps extends the shoulder as well as the elbow, impeding the coordination of the shoulder and elbow joints. Finally, the coracobrachialis produces shoulder flexion that was found to be disadvantageous when coupled to the elbow flexion actions of the other muscles innervated by the musculocutaneous nerve (biceps, brachialis).

Electrode selection in this study was based on a model of a generic high-tetraplegia subject. Even though the model parameters, some of which were measured from cadaver studies (skeletal dimensions and masses, muscle attachment sites) and some of which were assumed (e.g., 50\% maximum forces in paralyzed but stimulated muscles), do not correspond perfectly to those of a particular FES participant, meaningful conclusions can still be reached about the muscles that would be most effective in restoring movements to paralyzed individuals. 
Using anatomical and physiological information from a particular individual, this technique could result in a neuroprosthetic system that is further customized to the person's specific needs. For example, magnetic resonance imaging techniques could be used to estimate the maximum forces expected from paralyzed muscles [22]. In addition, the simulations could focus on a different set of movements needed to achieve the particular functional goals of a specific person. In cases of extensive muscle denervation, a mobile arm support could be simulated, added to the arm model, and then used in simulation to investigate the effect of combined FES and orthotic support against gravity.

\section{CONCLUSIONS}

We have presented a systematic approach to the selection of muscle and nerve-cuff electrodes for use in neuroprosthetic systems that considers functional goals, hardware limitations, muscle and nerve anatomy, and surgical feasibility. Nerve-cuff placement and selectivity were found to be important factors in the determination of predicted function, as well as the choice of which nerves and muscles to target. The use of musculoskeletal models in this way can thus greatly facilitate the development of neuroprosthetic systems by quantifying the importance of various muscles on different movements and allowing us to appropriately allocate the available stimulating electrodes without the need for time-consuming trial-and-error experiments. As FES hardware becomes more sophisticated and the applications become more ambitious, the use of customizable musculoskeletal models will allow us to make informed design decisions in order to maximize the functional gain for prospective FES users.

\section{ACKNOWLEDGMENTS}

\author{
Author Contributions: \\ Performed simulations: D. Blana, J. G. Hincapie. \\ Analyzed data: D. Blana, J. G. Hincapie, E. K. Chadwick. \\ Wrote manuscript: D. Blana. \\ Edited manuscript: E. K. Chadwick, R. F. Kirsch. \\ Supervised project: R. F. Kirsch.
}

Financial Disclosures: The authors have declared that no competing interests exist.
Funding/Support: This material was based on work supported by the National Institutes of Health/National Institute of Neurological Disorders and Stroke (contracts N01-NS-1-2333 and N01-NS-5-2365).

Additional Contributions: Juan G. Hincapie is now with Boston Scientific, Minnesota. Edward K. Chadwick is now with Institute for Science and Technology in Medicine, Keele University, United Kingdom.

\section{REFERENCES}

1. Crago PE, Memberg WD, Usey MK, Keith MW, Kirsch RF, Chapman GJ, Katorgi MA, Perreault EJ. An elbow extension neuroprosthesis for individuals with tetraplegia. IEEE Trans Rehabil Eng. 1998;6(1):1-6. [PMID:9535517]

2. Keith MW, Peckham PH, Thrope GB, Buckett JR, Stroh KC, Menger V. Functional neuromuscular stimulation neuroprostheses for the tetraplegic hand. Clin Orthop Relat Res. 1988;233(233):25-33. [PMID:3042233]

3. Kilgore KL, Peckham PH, Thrope GB, Keith MW, GallaherStone KA. Synthesis of hand grasp using functional neuromuscular stimulation. IEEE Trans Biomed Eng. 1989;36(7): 761-70. [PMID:2787284] http://dx.doi.org/10.1109/10.32109

4. Davis JA Jr, Triolo RJ, Uhlir JP, Bhadra N, Lissy DA, Nandurkar S, Marsolais EB. Surgical technique for installing an eight-channel neuroprosthesis for standing. Clin Orthop Relat Res. 2001;385(385):237-52. [PMID:11302320] http://dx.doi.org/10.1097/00003086-200104000-00035

5. Nathan RH. An FNS-based system for generating upper limb function in the C4 quadriplegic. Med Biol Eng Comput. 1989;27(6):549-56. http://dx.doi.org/10.1007/BF02441635

6. Nathan RH, Ohry A. Upper limb functions regained in quadriplegia: a hybrid computerized neuromuscular stimulation system. Arch Phys Med Rehabil. 1990;71(6):415-21. [PMID:2334287]

7. Hoshimiya N, Naito A, Yajima M, Handa Y. A multichannel FES system for the restoration of motor functions in high spinal cord injury patients: a respiration-controlled system for multijoint upper extremity. IEEE Trans Biomed Eng. 1989;36(7):754-60. [PMID:2787283] http://dx.doi.org/10.1109/10.32108

8. van der Helm FC. A finite element musculoskeletal model of the shoulder mechanism. J Biomech. 1994;27(5):551-69. [PMID:8027090] http://dx.doi.org/10.1016/0021-9290(94)90065-5

9. Kirsch RF, Acosta AM, van der Helm FC, Rotteveel RJ, Cash LA. Model-based development of neuroprostheses for restoring proximal arm function. J Rehabil Res Dev. 2001;38(6):619-26. [PMID:11767969]

10. Hincapie JG, Blana D, Chadwick EK, Kirsch RF. Musculoskeletal model-guided, customizable selection of shoulder 
and elbow muscles for a C5 SCI neuroprosthesis. IEEE Trans Neural Syst Rehabil Eng. 2008;16(3):255-63.

[PMID:18586604]

http://dx.doi.org/10.1109/TNSRE.2008.922681

11. Kilgore KL, Peckham PH, Keith MW, Montague FW, Hart RL, Gazdik MM, Bryden AM, Snyder SA, Stage TG. Durability of implanted electrodes and leads in an upper-limb neuroprosthesis. J Rehabil Res Dev. 2003;40(6):457-68. [PMID:15077658] http://dx.doi.org/10.1682/JRRD.2003.11.0457

12. Memberg WD, Peckham PH, Keith MW. A surgicallyimplanted intramuscular electrode for an implantable neuromuscular stimulation system. IEEE Trans Rehabil Eng. 1994;2(2):80-91. http://dx.doi.org/10.1109/86.313149

13. Naples GG, Mortimer JT, Scheiner A, Sweeney JD. A spiral nerve cuff electrode for peripheral nerve stimulation. IEEE Trans Biomed Eng. 1988;35(11):905-16. [PMID:3198136] http://dx.doi.org/10.1109/10.8670

14. Grill WM Jr, Mortimer JT. Quantification of recruitment properties of multiple contact cuff electrodes. IEEE Trans Rehabil Eng. 1996;4(2):49-62. [PMID:8798072] http://dx.doi.org/10.1109/86.506402

15. Polasek KH, Hoyen HA, Keith MW, Kirsch RF, Tyler DJ. Stimulation stability and selectivity of chronically implanted multicontact nerve cuff electrodes in the human upper extremity. IEEE Trans Neural Syst Rehabil Eng. 2009;17(5):428-37. [PMID:19775987] http://dx.doi.org/10.1109/TNSRE.2009.2032603

16. Hart RL, Bhadra N, Montague FW, Kilgore KL, Peckham $\mathrm{PH}$. Design and testing of an advanced implantable neuroprosthesis with myoelectric control. IEEE Trans Neural Syst Rehabil Eng. 2011;19(1):45-53.

17. Klein Breteler MD, Spoor CW, Van der Helm FC. Measuring muscle and joint geometry parameters of a shoulder for modeling purposes. J Biomech. 1999;32(11):1191-97. [PMID:10541069] http://dx.doi.org/10.1016/S0021-9290(99)00122-0
18. Blana D, Hincapie JG, Chadwick EK, Kirsch RF. A musculoskeletal model of the upper extremity for use in the development of neuroprosthetic systems. J Biomech. 2008;41(8):1714-21. [PMID:18420213] http://dx.doi.org/10.1016/j.jbiomech.2008.03.001

19. Heilman BP, Audu ML, Kirsch RF, Triolo RJ. Selection of an optimal muscle set for a 16-channel standing neuroprosthesis using a human musculoskeletal model. J Rehabil Res Dev. 2006;43(2):273-86. [PMID:16847793] http://dx.doi.org/10.1682/JRRD.2005.04.0072

20. Praagman M, Chadwick EK, van der Helm FC, Veeger HE. The relationship between two different mechanical cost functions and muscle oxygen consumption. J Biomech. 2006;39(4):758-65. [PMID:16439246] http://dx.doi.org/10.1016/j.jbiomech.2004.11.034

21. de Groot JH, Brand R. A three-dimensional regression model of the shoulder rhythm. Clin Biomech (Bristol, Avon). 2001;16(9):735-43. [PMID:11714550] http://dx.doi.org/10.1016/S0268-0033(01)00065-1

22. Fukunaga T, Roy RR, Shellock FG, Hodgson JA, Day MK, Lee PL, Kwong-Fu H, Edgerton VR. Physiological crosssectional area of human leg muscles based on magnetic resonance imaging. J Orthopaedic Res. 1992;10(6):928-34.

Submitted for publication February 21, 2012. Accepted in revised form July 25, 2012.

This article and any supplementary material should be cited as follows:

Blana D, Hincapie JG, Chadwick EK, Kirsch RF. Selection of muscle and nerve-cuff electrodes for neuroprostheses using customizable musculoskeletal model. J Rehabil Res Dev. 2013;50(3):395-408.

http://dx.doi.org/10.1682/JRRD.2012.02.0034

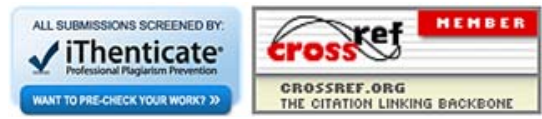


